\title{
Especie nueva de Dolichoderus (Hymenoptera: Formicidae) de Puerto Vallarta, Jalisco y nuevos registros para México
}

\section{A new species of Dolichoderus (Hymenoptera: Formicidae) from Puerto Vallarta, Jalisco, and new records for Mexico}

\author{
José Luis Ortega-De Santiago y Miguel Vásquez-Bolaños ${ }^{\bigotimes}$ \\ Entomología, Centro de Estudios en Zoología, Departamento de Botánica y Zoología, Centro Universitario de Ciencias Biológicas y Agropecuarias, \\ Universidad de Guadalajara. Apartado postal 134, 45100 Zapopan, Jalisco, México. \\ \vvb14145@hotmail.com
}

\begin{abstract}
Resumen. Se describe Dolichoderus tridentanodus sp. nov. recolectada en Puerto Vallarta, Jalisco, México, con base en obreras y que pertenece al complejo lugens. Esta especie se distingue de D. lugens por presentar 6 dientes en el margen interno de la mandíbula, 2 espinas dorsales en el propodeo, 2 protuberancias a los costados del pecíolo y 1 espina central; el cuerpo con abundante pubescencia de color dorado y cubierto por puntuaciones densas y marcadas; es negro, con mandíbulas, clípeo, antenas y patas de color amarillo. Se cita para los estados de Jalisco, Nayarit y San Luis Potosí. Se proporciona una clave para las especies de Dolichoderus en México, así como la distribución de las especies con nuevos registros para algunos estados.

Palabras clave: Dolichoderinae, complejo lugens, taxonomía alfa, Jalisco, Formicidae.

Abstract. We describe Dolichoderus tridentanodus sp. nov. collected in Puerto Vallarta, Jalisco, Mexico based on workers, which is placed in the lugens complex. This species can be distinguished from $D$. lugens by the presence of 6 teeth in the internal margin of the mandible, 2 spines on the dorsal face of the propodeum, 2 lateral projections on the petiole and 1central spine; body with abundant golden pubescence, densely and uniformly covered with coarse punctures; dark color, with yellow mandibles, clipeus, antenna and legs. This species is known for the states of Jalisco, Nayarit and San Luis Potosí. We provide a key for all the species of Dolichoderus in Mexico, and data of distribution with new records for some states.
\end{abstract}

Key words: Dolichoderinae, lugens complex, alpha taxonomy, Jalisco, Formicidae.

\section{Introducción}

Dolichoderus Lund pertenece a la tribu Dolichoderinii de la subfamilia Dolichoderinae (Cuezzo, 2003). Se conocen más de 183 especies en el mundo (Bolton et al., 2006) y para América se registran 64 (Ortiz y Fernández, 2011), agrupadas en complejos por tratarse de un grupo difícil taxonómicamente (Mackay, 1993). Estas hormigas son principalmente arborícolas y de amplia distribución en las regiones Neártica, Neotropical, Paleártica, Oriental y Australiana (Fisher, 2010), aunque la mayoría se encuentra en la región Neotropical (Ortiz y Fernández, 2011). Las hormigas pertenecientes al género se reconocen por la presencia de una expansión anterolateral en el hipostoma, mesoesternon expandido anteriormente, espinas o protuberancias en el propodeo; en vista dorsal, la línea superior del mesosoma se interrumpe por una constricción; además, presenta un

Recibido: 05 marzo 2012; aceptado: 14 mayo 2012 nodo peciolar relativamente grueso y abultado (Cuezzo, 2003; Shattuck, 1992).

En México se registran 4 complejos de los 12 conocidos para América, bispinosus, diversus, luges y quadripunctatus. Las especies del complejo bispinosus tienen la cabeza en forma de corazón, presentan espinas pronotales cortas (exceptuando D. mesonotalis) y 1 espina en el dorso del pecíolo; las reinas son muy similares, sólo se diferencian por el tamaño de las espinas, que son más pequeñas (Ortiz y Fernández, 2011; MacKay, 1993). De este complejo, está registrada para México $D$. bispinosus (Vásquez-Bolaños, 2011). El complejo diversus se caracterizan por presentar pelos suberectos en el escapo, la superficie dorsal del propodeo tiene un declive y usualmente está separada por una carina transversal y el nodo peciolar es muy angosto en vista lateral y raramente es bidentado, son relativamente más pequeñas, al menos las distribuidas de México a Brasil; los ángulos del propodeo están poco desarrollados y conectados con las carinas transversales; las reinas son similares a las 
obreras (Ortiz y Fernández, 2011; Mackay, 1993). De este complejo, hasta el momento se han registrado para México D. diversus, D. germaini y D. lutosus (VásquezBolaños, 2011). El complejo lugens se reconoce por las gruesas puntuaciones que cubren el cuerpo y su coloración negra con los apéndices castaños; el nodo peciolar grueso y redondeado, el propodeo no se extiende por encima del pecíolo y el mesonoto es más ancho que largo ( Ortiz y Fernández, 2011; Mackay, 1993). Este complejo sólo contiene una especie, D. lugens, registrada por primera vez para México en 1998 (Vásquez, 1998). Las especies del complejo quadripunctatus se caracterizan por que el propodeo que se extiende sobre el pecíolo y el pronoto carece de ángulos o espinas (MacKay, 1993). De este complejo, está registrada para México D. plagiatus (Vásquez-Bolaños, 2011).

En México están registradas 6 de las especies del género: D. bispinosus Olivier, 1792, para los estados mexicanos de Campeche, Chiapas, Quintana Roo, Tabasco, Veracruz y Yucatán; D. diversus Emery, 1894, para Quintana Roo y Tabasco; D. germaini Emery, 1894, sin localidad; D. lugens Emery, 1894, para Jalisco; D. lutosus Smith, 1858, para Chiapas, Quintana Roo, Tabasco y Veracruz y D. plagiatus Mayr, 1870, que sólo se conoce para Nuevo León (Vásquez-Bolaños, 2011).

\section{Materiales y métodos}

Los ejemplares fueron recolectados en la región sur de Puerto Vallarta, Jalisco. El municipio de Puerto Vallarta se localiza en la costa occidental del estado de Jalisco, entre los $20^{\circ} 27^{\prime} 00^{\prime \prime}$ y $20^{\circ} 59^{\prime} 00^{\prime \prime} \mathrm{N}, 104^{\circ} 55^{\prime} 00^{\prime \prime}$ y $105^{\circ} 33^{\prime} 00^{\prime \prime} \mathrm{O}$, en alturas que van desde 0 hasta 1 $600 \mathrm{~m}$. Limita al norte con el estado de Nayarit, al sur con el municipio de Cabo Corrientes y Talpa de Allende, al este con San Sebastián del Oeste y Mascota, y al oeste con el océano Pacífico. Tiene una extensión de 1 $300.67 \mathrm{~km}^{2}$; la mayor parte de la superficie está ocupada por zonas accidentadas, que forman parte de la sierra Madre Occidental. Cuenta con un clima semitropical y húmedo, temperatura máxima de $31^{\circ} \mathrm{C}$ en verano y mínima de $19^{\circ} \mathrm{C}$ en invierno; la media anual es de $25^{\circ}$ C. La temporada de lluvias abarca de mediados de junio a finales de agosto; la precipitación media anual es de $1417 \mathrm{~mm}$. Los vientos dominantes van en dirección suroeste y no se presentan heladas (SEGOB, 2010). La zona donde se realizaron las recolectas se encuentra cerca del río Palo María en las coordenadas 20³3'6" $\mathrm{N}, 105^{\circ} 16^{\prime} 0.9^{\prime \prime} \mathrm{O}$, con vegetación perturbada de bosque tropical subcaducifolio a una altitud de $10 \mathrm{~m}$. La recolecta se realizó de manera directa sobre la vegetación y con trampas de caída cebadas con atún.
Los ejemplares recolectados se examinaron con las claves que se incluyen en la revisión más reciente para el género (MacKay, 1993) y se compararon con los depositados en la Colección Entomológica de la Universidad de Guadalajara; se determinó que pertenecen al complejo lugens que hasta hoy sólo incluye 1 especie: D. lugens Emery. Los ejemplares examinados difieren significativamente de la especie conocida, por lo que se concluyó que pertenecen a una especie nueva, que aquí se describe.

Se midieron 14 ejemplares con un estereomicroscopio Carl-Zeiz SV40. Las medidas son las propuestas por MacKay (1993), en milímetros y con las siguientes abreviaturas: LC, longitud de la cabeza; AC, ancho de la cabeza; LO, longitud del ojo; LE, longitud del escapo; LW, longitud de Weber; LM, longitud del mesonoto; AM, ancho del mesonoto; AP, ancho del pecíolo; LP, longitud del pecíolo; IC, índice cefálico; IO, índice ocular; IE, índice del escapo; IM, índice del mesonoto.

\section{Descripción}

Dolichoderus tridentanodus sp. nov. Ortega-De Santiago y Vásquez-Bolaños (Figs. 1-3).

Holotipo (obrera). Medidas (mm)- LC 0.9; AC 0.95; LO 0.25; LE 1.0; LW 1.05; LM 1.5; AM 0.7; AP 0.35; LP 0.25; IC 105; IO 27; IE 111; IM 214. Mandíbulas con 6 dientes; clípeo en forma de trapecio, de color castaño claro; pronoto con ángulos marcados; metanoto con constricción y 2 lóbulos laterales; propodeo con 2 espinas pequeñas; pecíolo con 2 proyecciones laterales y 1 espina central, que se pueden presentar reducidas o gastadas pero siempre presentes; escultura en cabeza, mesosoma y pecíolo con puntos pronunciados; pilosidad formada por pelos más largos que el ancho del escapo cubriendo todo el cuerpo; pelos erectos en el dorso de la cabeza y gáster más gruesos que el resto, pelos decumbentes en el dorso del gáster abundantes. Paratipos (once individuos medidos; obreras).- LC 0.8-1.0; AC 0.8-1.05; LO 0.025; LE 0.9-1.0; LW 1.15-1.4; LM 1.45-1.6; AM 0.6-0.75; AP 0.25-0.35; LP 0.25-0.3; IC 94.7-112; IO 2.5-3.1; IE 100112.5; IM 200-242.

Obrera. Mandíbulas con 6 dientes en el margen interno; clípeo diferenciado en forma de trapecio de color café claro a castaño; pronoto con ángulos marcados; metanoto con constricción y 2 lóbulos laterales; propodeo con un par de espinas pequeñas; pecíolo con 2 proyecciones laterales y una espina central; cabeza, mesosoma y pecíolo con ornamentación a manera de puntos pronunciados; pelos en el dorso del cuerpo más largos que el ancho del escapo; pelos abundantes y erectos en el dorso de la cabeza y gáster, más gruesos que en el resto del cuerpo; 


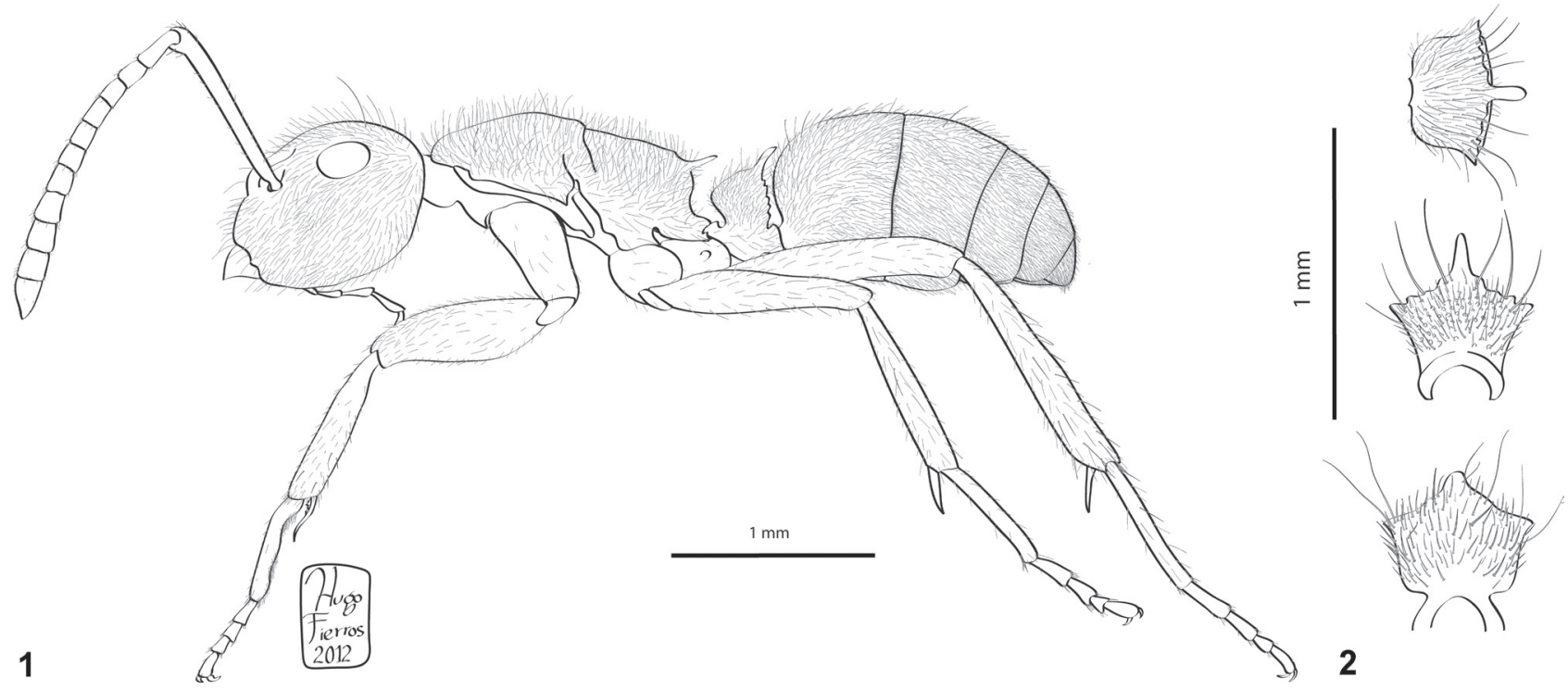

Figuras 1-2. Dolichoderus tridentanodus sp. nov., obrera. 1, vista lateral; 2, vista frontal del pecíolo.

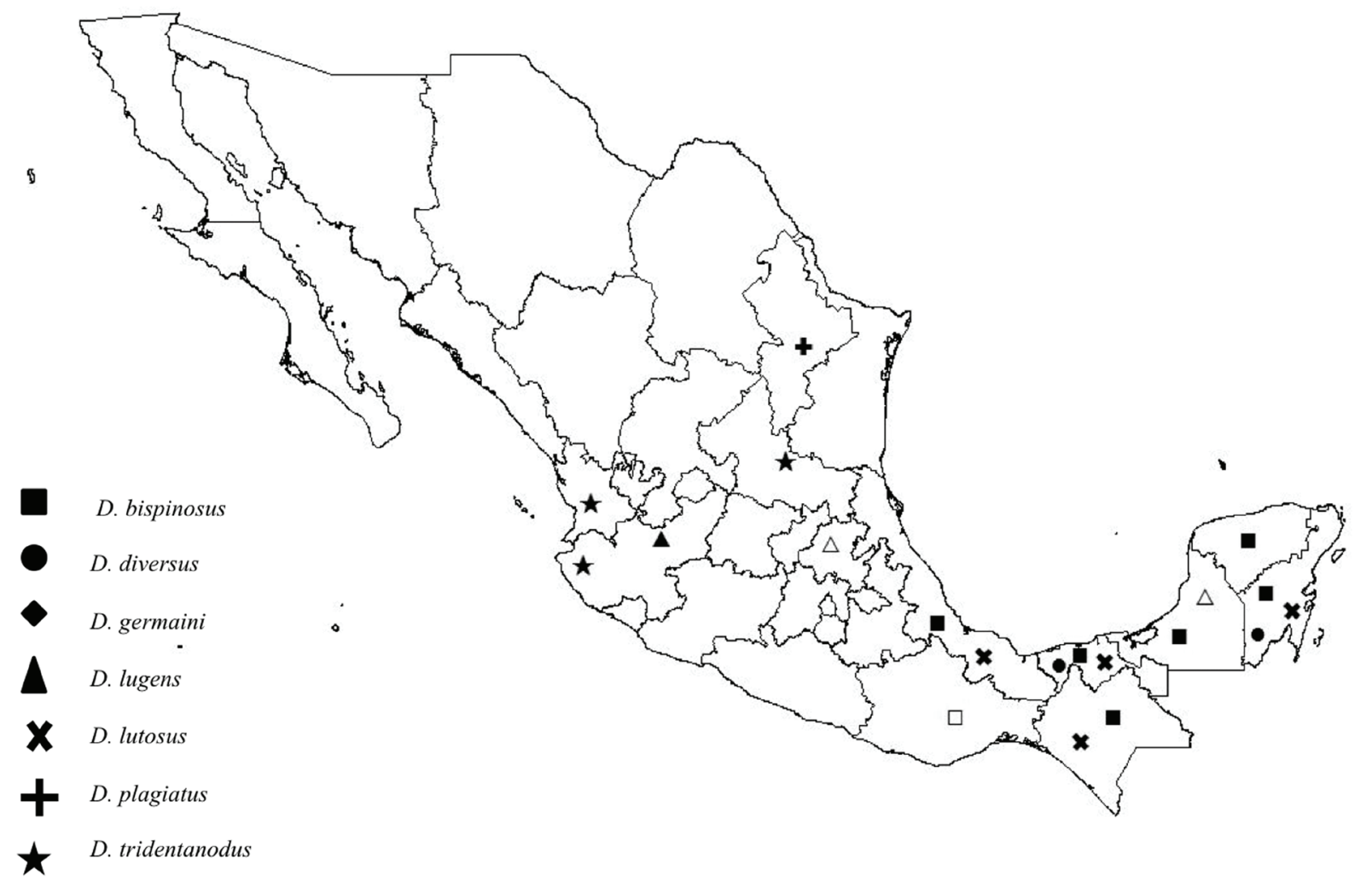

Figura 3. Mapa de distribución de las especies conocidas de Dolichoderus en México. Símbolos oscuros, localidades conocidas; símbolos claros, registros nuevos. 
y pelos decumbentes abundantes en el dorso del gáster (Figs. 1, 2).

\section{Resumen taxonómico}

Material examinado. Serie tipo. Holotipo, obrera etiquetada: México, Jalisco, Puerto Vallarta, Palo María, río Palo María, BTSCpert, 10m., 203' 6" N, 105¹6'0.9" O, 3.I.2009, colecta directa, A. Meza-López col. Paratipos, 11 obreras etiquetadas como sigue: 5, misma información, 6.VI.2009; 1, misma información, 2.V.2009, trampa de caída con atún; 2, misma información, 2.V.2009; 1, misma información, 5.IX.2009; 2, misma información, 5.XII.2009.

Material adicional. Una obrera etiquetada como sigue: México, Nayarit, Sayulita, 23.V.2011, $0 \mathrm{~m}$, palmardunas costeras, $20^{\circ} 52^{\prime} 19.03^{\prime \prime} \mathrm{N}, 105^{\circ} 20^{\prime} 48.8^{\prime \prime} \mathrm{O}, \mathrm{P}$. Montiel-Moncayo col.; 1 obrera misma información: M. A. García-Martínez col.; 1 obrera etiquetada como sigue: México, San Luis Potosí, Tamasopo, Cascadas de Tamasopo, BTSC, 21 $56^{\prime} 3.4^{\prime \prime}$ N, 99²3'7.1” O, 475 m., 30.VII.2003, M. Vásquez col.

El material se encuentra depositado en la Colección Entomológica del Centro de Estudios en Zoología de la Universidad de Guadalajara, Jalisco, México, CZUG (holotipo y 8 paratipos); Colección de Hormigas del Laboratorio de Ecología y Sistemática de Microartrópodos de la Universidad Nacional Autónoma de México, Querétaro, México, LESM (1 paratipo); Colección de Insectos del Instituto de Ecología, A. C., Veracruz, México, INECOL (1 paratipo); Colección de hormigas de William y Emma MacKay, Texas, Estados Unidos de América, CWEM (1 paratipo).

Macho y reina: desconocidos.

Etimología: se le da el nombre de tridentanodus por los 3 dientes que presenta en el nodo del pecíolo, 2 laterales y 1 central.

\section{Comentarios taxonómicos}

Los ejemplares (obreras) se recolectaron de manera directa sobre vegetación y 1 con trampa de caída, cebada con atún, en sitios húmedos o cerca de cuerpos de agua. Se encuentran en lugares de baja altitud, menor a $50 \mathrm{~m}$, aunque el ejemplar de San Luis Potosí se colectó a casi $500 \mathrm{~m}$.

Adicionalmente se revisó material que corresponde a otras especies del Dolichoderus depositado en varias colecciones: Colección Entomológica de la Universidad de Guadalajara (CZUG); Colección de Hormigas de la Universidad de Quintana Roo (CHUQROO); Colección Santiago Niño Maldonado (SNM). Complejo lugens, D. lugens Emery, 1894: 1 obrera etiquetada: México, Jalisco, Zapopan, ex Hacienda del Lazo, BTCpert., 1150 m., M. Vásquez col. 16.XI.1996; 20 obreras etiquetadas: México, Jalisco, Tequila, camino a La Toma, BTSCpert.,
2.XI.2010, M. Vásquez-Bolaños col.; 2 obreras etiquetadas: México, Jalisco, Chamela, La Huerta, Estación Científica, BTC, 24.VII.2010, 19³1'26.5”' $\mathrm{N}, 105^{\circ} 2^{\prime} 13.2$ " O, $37 \mathrm{~m}, \mathrm{M}$. Vásquez-Bolaños col.; 2 obreras etiquetadas: México, Campeche, Xpujil, Reseva de Calakmul, $54 \mathrm{~km}$ al N. de Xpujil, BTSP, 11.IX.1999, $264 \mathrm{~m}, 18^{\circ} 51^{\prime} 31.57^{\prime \prime} \mathrm{N}, 89^{\circ} 31^{\prime} 44.90^{\prime \prime} \mathrm{O}$, J. A. RodríguezGarza col.; 3 obreras etiquetadas como sigue: México, Hidalgo, Huazalingo, Zoquicualoya, 560m., 2059'33.1" N, 98²8’3.4” O, 10.III.2010, I. Hernández Pérez y L. Ramírez Martínez cols.; 1 obrera etiquetada como sigue: mismos datos, Tepemaxac, 31.III.2010, 780m., $21^{\circ} 1^{\prime} 51.3$ " N, 98 $29^{\prime} 28.4$ " O; 1 obrera etiquetada como sigue: mismos datos, Santa María, 5.IV.2011, 525m., $20^{\circ} 59^{\prime} 52.9^{\prime}$ N, $98^{\circ} 28^{\prime} 8.9^{\prime \prime} \mathrm{O}$; 1 obrera etiquetada como sigue: mismos datos, 7.IV.2011; 1 obrera etiquetada como sigue: mismos datos, San Francisco, 23.VI.2011, $560 \mathrm{~m} ., 21^{\circ} 0^{\prime} 54.8^{\prime \prime} \mathrm{N}, 98^{\circ} 29^{\prime} 9^{\prime \prime} \mathrm{O} ; 3$ obreras etiquetadas como sigue: mismos datos, San Pedro, 396m., 2057'2.1" N, 98³’33.6” O, 16.VIII.2009. Complejo bispinosus, D. bispinosus (Olivier, 1792): 1 obrera con los siguientes datos de etiqueta: México, Chiapas, Tuxtla Gutiérrez, ZOOMAT, BTSC, $16^{\circ} 43^{\prime} 22^{\prime \prime}$ N , 9305' $52^{\prime}$ ' O, $720 \mathrm{~m}$., 1.XII.2008, J. Reynoso col.; 5 obreras etiquetadas como sigue: México, Quintana Roo, Otón P. Blanco, Chetumal, Reserva de la Biosfera San Felipe Bacalar, 6.IX.2011, $18 \mathrm{~m}, 18^{\circ} 46^{\prime} 19.9^{\prime \prime} \mathrm{N}, 88^{\circ} 24^{\prime} 53.3^{\prime \prime} \mathrm{O}$, M. VásquezBolaños col.; 1 obrera y 1 reina: México, Campeche, Calakmul, 7.VII.2011, 1759'48.3” N, 8944'11.5” O, 234m., G. Contreras col.; 6 obreras México, Quintana Roo, Felipe Carrillo Puerto, Sian Ka'an, 1.VIII.2011, $18^{\circ} 59^{\prime} 31.3^{\prime \prime} \mathrm{N}, 87^{\circ} 43^{\prime} 50.1^{\prime \prime} \mathrm{O}, 2$ m., G. Contreras col.; 7 obreras etiquetadas: México, Oaxaca, Valle Nacional, Km. 56 Carretera Oaxaca-Valle Nacional, BG, 577m, $17^{\circ} 43^{\prime} 50.3^{\prime}$ " N, 96¹9'47.6” O, M. Vásquez-Bolaños col. Complejo diversus, D. diversus Emery, 1894: 2 obreras etiquetadas como sigue: México, Quintana Roo, Bacalar, Reserva de la Biosfera San Felipe Bacalar, 1846'19.9" $\mathrm{N}, 8^{\circ} 24^{\prime} 53.3^{\prime \prime}$ O, 18m., 6.IX.2011, M. Vásquez-Bolaños col.; 2 obreras etiquetadas como sigue: México, Quintana Roo, Othón P. Blanco, Subteniente López, río Hondo, $5 \mathrm{~m}$ manglar, SE-6-D-N, H. Suárez col.; D. lutosus (Smith, 1858): 3 obreras etiquetadas como sigue: México, Quintana Roo, Othón P. Blanco, Río Hondo, 3m manglar, DK-4-V, H. Suárez col. (Fig. 3).

Dolichoderus tridentanodus sp. nov. puede distinguirse de $D$. lugens por presentar 6 dientes del borde interno de la mandíbula ( 8 en $D$. lugens), 1 par de espinas en el propodeo, 2 protuberancias a los costados del pecíolo y 1 espina central (espinas y protuberancias ausentes en $D$. lugens), abundante pubescencia (ausente en $D$. lugens) de color dorado y marcadas puntuaciones en todo el cuerpo, 
así como las mandíbulas y el clípeo de color amarillo (puntuaciones y mandíbulas amarillas ausentes en D. lugens).

Se han citado 7 especies de Dolichoderus para México. Se registra por primera vez $D$. lugens para los estados de Campeche e Hidalgo; así como D. bispinosus para el estado de Oaxaca (Fig. 3). Las especies se distribuyen por debajo de los $1150 \mathrm{~m}$, en vegetación de tipo caducifolio a perennifolio, en lugares húmedos o cerca de cuerpos de agua.

Lista de las especies de Dolichoderus citadas para México: complejo bispinosus, D. bispinosus (Olivier, 1792); complejo diversus, D. diversus Emery, 1894, D. germaini Emery, 1894, D. lutosus (Smith, 1858); complejo lugens, D. lugens Emery, 1894, D. tridentanodus sp. nov.; complejo quadripunctatus, D. plagiatus (Mayr, 1870).

\section{Agradecimientos}

A Abraham Meza López, por la recolecta de los ejemplares que corresponden a la serie tipo y a Pablo Montiel Moncayo y Miguel Ángel García Martínez, por la recolecta de los ejemplares adicionales. A Juan Antonio Rodríguez Garza (Universidad de Quintana Roo) y Santiago Niño Maldonado (Universidad Autónoma de Tamaulipas), por permitir revisar sus respectivas colecciones. A Hugo Eduardo Fierros López (Universidad de Guadalajara), por la elaboración de los esquemas, a Marco Antonio Carrasco Ortiz, por la elaboración del mapa. Al personal de la Colección Entomológica CZUG, por el apoyo brindado. brindado. A Fabiana Cuezzo y a Steven O. Shattuck por la aportación de literatura.

Clave para las especies de Dolichoderus de México

1. Pronoto con un par de espinas (complejo bispinosus); sur de México: Campeche, Chiapas, Oaxaca, Quintana Roo, Tabasco, Veracruz y Yucatán ..... D. bispinosus

1'. Pronoto sin espinas .2

2. Propodeo desarrollado por encima del pecíolo (complejo quadripunctatus); norte de México: Nuevo León...D. plagiatus

2'. Propodeo no desarrollado por encima del pecíolo ...3

3. Pecíolo grueso y redondeado; color oscuro en el cuerpo con mandíbulas, antenas y patas claras (complejo lugens); Campeche, Hidalgo, Jalisco, Nayarit y San Luis Potosí...... ...4 3'. Pecíolo delgado y no redondeado; color uniforme en todo el cuerpo (complejo diversus); Chiapas, Quintana Roo, Tabasco y Veracruz ...... D. lugens 4. Propodeo y pecíolo sin espinas; Campeche, Jalisco e Hidalgo. D. tridentanodus sp. nov. 4'. Propodeo y pecíolo con espinas; Jalisco, Nayarit y San Luis Potosí...... D. tridentanodus sp. nov.

5. Escapo y tibias sin pilosidad; Chiapas, Quintana Roo, Tabasco y Veracruz.

5'. Escapo y tibias con pilosidad D. diversus

6. Superficie dorsal, laterales y posterior del propodeo redondeada; Quintana Roo y Tabasco. D. germaini

\section{Literatura citada}

Bolton, B., G. Alpert, P. S. Ward y P. Naskrecki. 2006. Bolton's catalogue of ants of the world: 1758-2005. Harvard University Press, Cambridge, Massachusetts. CD.

Cuezzo, F. 2003. Subfamilia Dolichoderinae. In Introducción a las hormigas de la región neotropical, F. Fernández (ed.). Instituto de Investigación de Recursos Biológicos Alexander van Humboldt, Bogotá. p. 291-298.

Fisher, B. L. 2010. Biogeography. In Ant ecology, L. Lanch, C. L. Parr y L. Abbott (eds.). Oxford University Press, New York. p. 18-37.

MacKay, W. P. 1993. A review of the New World ants of the genus Dolichoderus (Hymenoptera: Fomicidae). Sociobiology 22:1148.

Ortiz, C. M. y F. Fernández. 2011. Hormigas del género Dolichoderus Lund (Formicidae: Dolichoderinae) en Colombia. III Monografías de Fauna de Colombia. Universidad
Nacional de Colombia, Bogotá. 118 p.

SEGOB (Gobierno del Estado de Jalisco). 2010. Los municipios de Jalisco. Colección Enciclopedia de los Municipios de México. Instituto para el Federalismo y el Desarrollo Municipal (INAFED)-Secretaría de Gobernación / Gobierno del Estado de Jalisco, Guadalajara. http://www.inafed.gob.

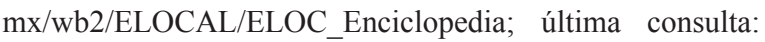
18.X.2011.

Shattuck, S. O. 1992. Generic revision of the ant subfamily Dolichoderinae (Hymenoptera: Formicidae). Sociobiology 21:1-184.

Vásquez, B., M. 1998. Hormigas (Hymenoptera: Fomicidae) colectadas en necrotrampas, en tres localidades de Jalisco, México. Tesis, División de Ciencias Biológicas y Ambientales, Universidad de Guadalajara, Zapopan, Jalisco. 43 p.

Vásquez-Bolaños, M. 2011. Lista de especies de hormigas (Hymenoptera: Formicidae) para México. Dugesiana18:95-133. 